\title{
The Bloodgen Project of the European Union, 2003-2009
}

\author{
Neil D. Avent ${ }^{\mathrm{a}} \quad$ Antonio Martinez ${ }^{\mathrm{b}} \quad$ Willy A. Flegel ${ }^{\mathrm{c}}$ Martin L. Olsson ${ }^{\mathrm{d}}$ Marion L. Scott
}

Núria Nogués ${ }^{f} \quad$ Martin Písăcka $^{g} \quad$ Geoff L. Daniels ${ }^{e} \quad$ Eduardo Muñiz-Diaz $^{f}$ Tracey E. Madgett $^{a}$

Jill R. Storry ${ }^{d}$ Sigrid Beiboer $^{\text {h }}$ Petra M. Maaskant-van Wijk ${ }^{\mathrm{h}}$ Inge von Zabern ${ }^{\mathrm{c}}$

Elisa Jiménez ${ }^{\mathrm{b}}$ Diego Tejedor ${ }^{\mathrm{b}}$ Mónica López $^{\mathrm{b}}$ Emma Camacho $^{\mathrm{f}}$ Goedele Cheroutre $^{\mathrm{h}}$ Anita Hacker ${ }^{c}$ Pavel Jinoch ${ }^{g}$ Irena Svobodova ${ }^{g}$ Ellen van der Schoot ${ }^{\text {h }}$ Masja de Haas ${ }^{\mathrm{h}}$

\footnotetext{
${ }^{a}$ Centre for Research in Biomedicine and Bristol Genomics Research Institute, Faculty of Applied Sciences,

University of the West of England, Bristol, UK

${ }^{\mathrm{b}}$ Progenika Biopharma S.A., Derio, Spain

'Institut Transfusionsmedizin, Universitätsklinikum and Institut Klinische Transfusionsmedizin und Immungenetik, Ulm, Germany

${ }^{\mathrm{d}}$ Blood Centre, University Hospital Blood Centre, Lund, Sweden

${ }^{e}$ Bristol Institute for Transfusion Sciences, Bristol, UK

${ }^{f}$ Banc de Sang i Teixits (BST), Barcelona, Spain

${ }^{9}$ Institute of Haematology and Blood Transfusion (UHKT), Prague, Czech Republic

${ }^{\mathrm{h}}$ Sanquin Blood Supply Foundation, Amsterdam, The Netherlands
}

\section{Key Words}

BLOODchip · Blood groups · Blood group antigens

\section{Summary}

The Bloodgen project was funded by the European Commission between 2003 and 2006, and involved academic blood centres, universities, and Progenika Biopharma S.A., a commercial supplier of genotyping platforms that incorporate glass arrays. The project has led to the development of a commercially available product, BLOODchip, that can be used to comprehensively genotype an individual for all clinically significant blood groups. The intention of making this system available is that blood services and perhaps even hospital blood banks would be able to obtain extended information concerning the blood group of routine blood donors and vulnerable patient groups. This may be of significant use in the current management of multi-transfused patients who become alloimmunised due to incomplete matching of blood groups. In the future it can be envisaged that better matching of donor-patient blood could be achieved by comprehensive genotyping of every blood donor, especially regular ones. This situation could even be extended to genotyping every individual at birth, which may prove to have significant long-term health economic benefits as it may be coupled with detection of inborn errors of metabolism.

\section{Schlüsselwörter}

BLOODchip · Blutgruppen · Blutgruppenantigene

\section{Zusammenfassung}

Das Bloodgen-Projekt wurde zwischen 2003 und 2006 von der Europäischen Kommission gefördert und bestand aus akademischen Blutzentren, Universitäten und der Progenika Biopharma S.A., einem kommerziellen Anbieter von Genotypisierungsplattformen, die Glas-Arrays beinhalten. Das Projekt führte zur Entwicklung eines kommerziell erwerbbaren Produkt - BLOODchip -, mit dem eine Person für alle klinisch signifikanten Blutgruppen umfassend genotypisiert werden kann. Das System wurde kommerziell verfügbar gemacht, um Blutspendeeinrichtungen und möglicherweise sogar Krankenhausblutbanken in die Lage zu versetzen, umfangreiche Informationen bezüglich der Blutgruppe von Dauerspendern und gefährdeten Patientengruppen zu generieren. Dies könnte von besonderer Bedeutung für das aktuelle Management von multitransfundierten Patienten sein, die aufgrund eines unvollständigen Blutgruppen-Matching alloimmunisiert wurden. Möglicherweise lässt sich zukünftig durch umfassende Genotypisierung aller Blutspender, besonders aber der Dauerspender, ein besseres Matching zwischen Spender- und Patientenblut erzielen. Möglicherweise wird es sogar eine Genotypisierung jeder Person bei seiner Geburt geben, durch die eine erhebliche Reduzierung der langfristigen Gesundheitskosten erzielt werden könnte, da sie mit der frühen Detektion von angeborenen Stoffwechseldefekten einhergeht.

\section{KARGER}

Fax +497614520714

Information@Karger.de

www.karger.com (c) 2009 S. Karger GmbH, Freiburg

Accessible online at:

www.karger.com/tmh 


\section{Introduction}

Throughout the 1990s all major blood group systems were defined at the level of the gene, and polymorphisms were deciphered for most blood group alleles [reviewed in 1-5]. These polymorphisms were mainly caused by single nucleotide polymorphisms (SNPs), but also, notably in the diverse $R H$ and $M N S$ systems, by gene conversions, duplications and, in the case of the Caucasian D-negative genotype, deletions. Most blood groups are dependent on polymorphic variation within protein structures (for example the $\mathrm{K} / \mathrm{k}$ (Kell) polymorphism is a SNP altering codon 183 methionine $(\mathrm{K})$ to threonine (k)) [6]. Throughout most of this decade this information remained largely an academic exercise with little direct application in transfusion medicine. There was one very important exception to this statement: genotyping for fetal $\mathrm{RhD}$ blood group for the management of haemolytic disease of the fetus and newborn (HDFN) was the first real clinical application of this information [7-11]. Later this methodology was applied to a number of other blood groups that are implicated in HDFN [12, 13], and these assays were initially applied to fetal material obtained by amniocentesis or chorionic villus (CV) sampling, which was spare material normally disposed of by the now obsolete Liley technique for the prediction of severity of HDFN [14]. In the late 1990s / early 2000s the emphasis was switched to using maternal plasma instead of invasively sampled fetal material [15-17], which eliminated any procedurally related risk (approximately $1 \%$ of fetuses spontaneously abort during the amniocentesis procedure). Non-invasive testing for fetal $R H D$ blood group genotype is now widespread and has led to the elimination of amniocentesis and CV sampling for assessment of HDFN [18-20].

Fetal genotyping for blood group status has remained the main usage of DNA-based typing in Europe although donor genotyping is becoming more commonplace in North America. With the evolution of high-throughput genotyping platforms, especially glass and bead array approaches, the feasibility of utilising such systems to enable closer matching of donors and patients was quickly realised [21]. Several projects were initiated in the early 2000 s as feasibility studies using these genotyping systems [reviewed in 22]. This review describes the efforts of the Bloodgen consortium ( $w w w$. bloodgen.com) which developed a glass array capable to genotyping over 116 blood group-specific SNPs (BLOODchip version 1.0). Since completion of the project in September 2006 BLOODchip version 1.0 has been CE-marked for $\mathrm{RhCE}$, Kell as required in current directives from the European Commission (EC). Version 1.0 has the capacity to detect all major $A B O, R H D, R H C E, M N S, K E L, F Y, J K$, $D O, D I$, and $C O$ alleles. Further developments of BLOODchip include version 2 (addition of all clinically relevant $H P A$ alleles) and version 3 (new RHCE and RHD alleles, plus $L W, L U$ alleles, and additional $J K$ alleles). BLOOD- chip will shortly be CE-marked for RhD diagnostic use, but at the present time there are no plans to $\mathrm{CE}$ mark for $\mathrm{ABO}$ diagnostic use, remaining as a research tool. This is because of the genetic complexity of $A B O$ alleles, and the potential risk that genotyping may mis-score an ABO blood unit. Nevertheless, we are confident that with extensive use and resultant determination of the majority of $A B O$ alleles, blood group genotyping may prove as robust as $\mathrm{ABO}$ serological testing and may replace it in routine use.

\section{Technical Background}

It is not the intention of this review to give a thorough review of the technology that supports the BLOODchip platform as these have been described in some detail before [22, 23]. In brief, the BLOODchip platform requires a standard approach for DNA extraction, followed by PCR amplification of DNA containing the SNPs responsible for blood group polymorphisms by a dedicated series of three multiplex (MPX) PCRs. The PCR products are then fragmented, labelled, and hybridised to a glass array containing multiple copies of probes corresponding to each paired allele. Detection of binding to each probe is then achieved using a standard laser array scanner. Then a comparison of strength of binding of the labelled PCR products to each probe is made using bespoke software. The BLOODchip system software then provides an output of genotype and predicted serological phenotype. The predicted phenotype is especially sensitive when considering variant $\mathrm{Rh}$ phenotypes, especially partial D. This was a major activity within the Bloodgen project - to produce a viable genotyping platform that is able to correctly predict unusual Rh phenotypes, the most complex of blood groups. After DNA extraction, approximately 6-8 $\mathrm{h}$ processing (PCR, labelling, fragmentation, hybridisation, and data interpretation) is required before the genotype is determined. For this reason, BLOODchip is not intended for use in emergency situations although in the future large banks of genotyped blood may significantly aid electronic cross-matching.

\section{Bloodgen Workpackage Structure}

The workload of the project was carried out in designated workpackages. Workpackage (WP) 1 concerned the fabrication of arrays and involved the design of probes complementary to the target DNAs containing the blood group-specific SNPs.

WP2 involved the development of fluoro-single-sequence primer assays. This WP was subsequently discontinued due to technical issues.

WP3 involved standardisation of DNA extraction and optimisation of MPX PCR assays. The MPX PCRs were designed 
following the generation of a list of required blood group SNPs that should populate the array. The labelling and fragmentation protocols were also optimised in this WP.

WP4 was a small-scale clinical trial. A biobank of extremely rare genomic DNA derived from individuals with $\mathrm{Rh}$ blood group phenotypes. These samples were then analysed with a prototype version of BLOODchip.

WP5 was a multi-centre clinical trial. Most Bloodgen participants then conducted a large-scale analysis of a cohort of genomic DNA samples obtained from blood donors, patients, newborns, and known weak D phenotype individuals.

Bloodgen formally finished with a dissemination event held at the International Society Blood Transfusion (ISBT) congress in Cape Town in September 2006. Progenika Biopharma (Derio, Spain) then continued to develop BLOODchip and completed the CE marking exercise with DNA samples provided by the consortium.

\section{Current Use Of Blood Group Genotyping and How BLOODchip May Assist These Situations}

Taking aside the aforementioned applications in fetal blood group genotyping, there are several instances where genotyping for blood group status has been applied. One major application has been in defining the blood group genotype in multitransfused patients where the presence of transfused blood makes it very difficult to ascertain the blood group of an individual by serology. DNA-based typing had been utilised for over a decade in the management of multi-transfused patients [24-26]. Conventional PCR-SSP (PCR with sequence-specific priming) approaches to type the recipient have been found to have low false-positive results, probably due to the fact that donor blood will be predominantly enucleated red blood cells devoid of DNA. One would anticipate that use of BLOODchip in such circumstances would also be unlikely to generate false-positive results. Clinical trials with BLOODchip and such samples are underway. Other studies have included the quality assurance of D-negative red cell units, to detect $\mathrm{RhD}$ variants that may not have been detected using conventional serological techniques. Molecular typing for $\mathrm{RhD}$ variants is much simpler than a serological investigation, which in theory would require a large battery of monoclonal anti-D and rare human polyclonal antisera to low-frequency antigens associated with partial D antigen (for example, BARC expressed in DVI; Tar on DVII red cells). Commercially available sequence-specific primer kits have been described [27], which test for a number of partial and weak D alleles, but by no means all. However, a major focus of the Bloodgen project was to produce a genotyping platform that could genotype the vast majority of $\mathrm{RhD}$ variants that are caused by either hybrid RHCE-RHD genes (for example DVI) [28-31] or point mutations (e.g. partial D, DNU [32] and weak D types [33, 34]). This is achieved by a combination of exon scanning, which is a process that entails amplifying every $R H D$ exon and BLOODchip detecting $R H D$ exons by having a specific probe set for each. Drop-out of RHD exons can then be readily detected, and a characteristic pattern corresponding to hybrid $R H D$ $R H C E$ genes can be deciphered and predicted. A probe set for each SNP causative of the vast majority of $\mathrm{RhD}$ variants (namely partial, weak, D-elute and D-negative) is present on BLOODchip version 1.0 and will be significantly extended on BLOODchip version 3.0.

Weakened Duffy b antigen expression was deciphered at the molecular level independently by three groups in 1998 [35-37]. This has allowed the determination of the Fy ${ }^{\text {bweak }}$ (or $\mathrm{Fy}^{\mathrm{x}}$ ) allele at the DNA level. Weakened Fy ${ }^{\mathrm{b}}$ expression can cause issues in the provision of reagent red cells that are of presumed genotype $F Y^{*} B / F Y^{*} B$, i.e. they are phenotypically $\mathrm{Fy}(\mathrm{a}-\mathrm{b}+)$. If an individual has the relatively common genotype $F Y^{*} B / F Y^{*} X$ then they would have substantially less $\mathrm{Fy}^{\mathrm{b}}$ antigen on their red cells than a homozygous $F Y^{*} B / F Y^{*} B$ individual. If the individual with weakened $\mathrm{Fy}^{\mathrm{b}}$ (namely presumed incorrectly to be $\mathrm{Fy}^{\mathrm{b}}$ homozygous) is selected as one of those for a red cell panel used to detect anti-Fy in patient sera, then there is suboptimal detection of this antibody. Such a scenario has been proven in a clinical situation [38]. True $F Y^{*} B$ homozygosity can be defined by genotyping to aid quality assurance of red cell panels.

\section{Performance of BLOODchip 1.0 in Clinical Trials}

Tables 1 and 2 review the performance of BLOODchip in CEmarking clinical trails and reveal the high degree of accuracy that can be achieved using this platform being significantly more accurate than serology. There are several examples of mis-typings by serology identified by BLOODchip that may lead to alloimmunisations which of course should be avoided, especially in vulnerable patient groups and individuals (for example multi-transfused patients and women of child-bearing age). Analysis of the 'non-Rh' blood groups reveals the accuracy of BLOODchip especially in FY and MNS blood groups. Rare MNS variants that are difficult to detect by serology and Fy ${ }^{\text {bweak }}$ antigen expression are major contributors to the better performance of BLOODchip. However, the performance of BLOODchip with RhD typing is outstanding and reveals several scenarios, which will be reviewed here, that could be avoided if genotyping replaces conventional serology.

\section{Mis-Typing Partial D Phenotype Samples as D-Positive by Serology}

BLOODchip identifies five such samples in the cohort of 3,000: 2 DVI, 1 DHMi, 1 DNU, and 1 DV individual. If these individuals were mis-typed as patients they could become alloimmunised by being transfused D-positive blood, or failing to receive prophylactic anti-D at the end of the pregnancy carrying a D-positive child. 
Table 1. CE marking exercise of BLOODchip, Rh CcEe, Kell, Duffy, Kidd, Dombrock, Diego and Colton antigens, versus serological typings, 1,000 genomic DNA samples (workpackage 5)

\begin{tabular}{|c|c|c|c|}
\hline Blood group antigen (system) & Serological testing & BLOODchip & Comments \\
\hline $\mathrm{Rh} \mathrm{C/c} \mathrm{(RH)}$ & $998 / 1,000$ & $999 / 1,000$ & \\
\hline $\mathrm{Rh} \mathrm{E} / \mathrm{e}(\mathrm{RH})$ & $997 / 1,000$ & $1,000 / 1,000$ & includes one typographical error \\
\hline $\mathrm{C}^{\mathrm{W}+}(\mathrm{RH})$ & $18^{*}$ & 28 & *serological confirmation of genotype \\
\hline $\mathrm{C}^{\mathrm{X}+}(\mathrm{RH})$ & $0 *$ & 2 & *serological confirmation of genotype \\
\hline $\mathrm{VS}+(\mathrm{RH})$ & $9 *$ & 15 & *serological confirmation of genotype \\
\hline $\mathrm{K} / \mathrm{k}(\mathrm{KEL})$ & $1,000 / 1,000$ & $1,000 / 1,000$ & \\
\hline $\mathrm{Kp} \mathrm{p}^{\mathrm{a}} / \mathrm{Kp}^{\mathrm{b}}(\mathrm{KEL})$ & $358 / 358$ & $357 / 358$ & \\
\hline $\mathrm{JS}^{\mathrm{a}} / \mathrm{JS}^{\mathrm{b}}(\mathrm{KEL})$ & $122 / 123$ & $123 / 123$ & \\
\hline $\mathrm{Jk}^{\mathrm{a}} / \mathrm{Jk}^{\mathrm{b}}(\mathrm{JK})$ & $596 / 597$ & $597 / 597$ & \\
\hline $\mathrm{Fy}^{\mathrm{a}} / \mathrm{Fy}^{\mathrm{b}} / \mathrm{Fy}(\mathrm{a}-\mathrm{b}-)(\mathrm{FY})$ & $498 / 506$ & $506 / 506$ & \\
\hline MN (MNS) & $425 / 455$ & $455 / 455$ & \\
\hline Ss (MNS) & $479 / 483$ & $483 / 483$ & \\
\hline $\mathrm{Di}^{\mathrm{a}} / \mathrm{Di}^{\mathrm{b}}(\mathrm{DI})$ & & $120 / 120$ & confirmed by DNA sequencing \\
\hline $\mathrm{Do}^{\mathrm{a}} / \mathrm{Do}^{\mathrm{b}} /(\mathrm{DO})$ & & $120 / 120$ & confirmed by DNA sequencing \\
\hline $\mathrm{Co}^{\mathrm{a}} / \mathrm{Co}^{\mathrm{b}}(\mathrm{CO})$ & $169 / 170$ & $170 / 170$ & \\
\hline
\end{tabular}

\section{Mis-Typing Partial D Phenotype Samples as Weak D by Serology}

Almost all (but definitely not all) [39] partial D phenotype samples have weakened expression of D antigen. Thus these may be typed serologically as weak D. However, if they are of known partial D type, then transfusion of D-positive blood to such individuals should be avoided, and in theory at least they should receive prophylactic anti-D during pregnancy as above. There has been some debate as to the classification of partial D and weak D [40], but any resultant nomenclature should communicate the risk of alloimmunisation to D antigen in each $\mathrm{RhD}$ variant as on the whole 'classical' weak D phenotypes are less prone to $\mathrm{RhD}$ alloimmunisation, whereas partial D are.

\section{Mis-Typing Partial D Phenotype as D-Negative by Serology} One such sample was found in the cohort of 3,000: a DIIIc phenotype individual. If such a sample is used for transfusion to a D-negative recipient, then there is the chance of producing anti-D. DIIIc phenotype individuals have relatively high $\mathrm{D}$ antigen site numbers [39], so its unclear why this was mistyped serologically but its identification within the donor cohort reveals it a risk that can be eliminated by mass-scale application of genotyping.

\section{Mis-Typing Weak D and D-Elute Phenotypes as D-Positive by Serology}

There were several examples of this detected by BLOODchip including weak D type 2, 3, 4, and 11. Particular weak D (and D-elute) phenotypes have known to become alloimmunised when transfused with D-positive blood [33, 41, 42], (weak D type 4.2 and 15 and D-elute RHD (IVS3+1g>a)) hence genotyping is able to identify such individuals, and their transfusion management can be adjusted accordingly. Interestingly, no samples amongst the cohort studied here were identified as weak D by BLOODchip but were RhD- by serology, indicat- ing the effectiveness of monoclonal anti-D reagents used by the consortium. In one study, a D-negative recipient received a unit of weak D type 2 red cells that had been mis-typed serologically as D-negative and as a consequence had become alloimmunised [41]

\section{Mis-Ttypings by BLOODchip}

There were two examples of BLOODchip scoring an individual as D-positive but was in fact detected as weak D by serology. The most likely outcome in this situation is that the individual carries an unknown RHD allele. BLOODchip has sufficient capacity to add all new $\mathrm{RhD}$ variants as they become described - indeed BLOODchip version 3.0 has 18 new RHD alleles added to it. It is hoped that these types of mis-scoring will be diminished if not eliminated when more widespread application of genotyping takes place.

\section{Future Use of Blood Group Genotyping}

The Bloodgen consortium have for many years been advocating the mass-scale application of genotyping as an alternative to blood group serology. Small-scale trials have already proven the effective superiority of genotyping-based approaches in a head-to-head comparison with serological investigations (see table 1). Where genotyping completely outperforms serology is in the analysis of variant $\mathrm{RhD}$ and $\mathrm{RhCE}$ individuals, the detection of Fy ${ }^{\text {bweak }}$, and unusual MNS variants. All of these situations are clinically relevant - partial D phenotype individuals can readily become immunised producing anti-D by either transfusion or pregnancy. RHCE (and MNS) variant alleles can cause significant problems in multi-transfused sickle cell patients [43. 44]. However, it must be stressed that at present BLOODchip typing has focussed largely on blood donors and to be truly effective typing of both patients and donors should be done to achieve most effective matching. 
Table 2. Performance of BLOODchip version 1.0 versus serology; RhD investigationsa

\begin{tabular}{|c|c|c|c|}
\hline \multicolumn{4}{|c|}{ Discordant results BLOODchip version 1.0 versus serology } \\
\hline Number & genotype (defined by BLOODchip) & BLOODchip & serology \\
\hline \multicolumn{4}{|c|}{ a) Data entry errors in serology (3) } \\
\hline 613 & DVI type II & partial D & $\mathrm{RhD}+$ \\
\hline 3693 & DVI type II & partial D & $\mathrm{RhD}+$ \\
\hline 3527 & weak D type 1 & weak D & $\mathrm{RhD}_{-}$ \\
\hline \multicolumn{4}{|c|}{ b) Partial D by BLOODchip version 1.0 and $R h D+$ by serology (5) } \\
\hline 1993 & DVI type IV & partial D & $\mathrm{RhD}+$ \\
\hline 2824 & DNU & partial D & $\mathrm{RhD}+$ \\
\hline 4154 & DVI type II & partial D & $\mathrm{RhD}+$ \\
\hline 4571 & DVa type 2 (Hus) / DV type 7 & partial D & $\mathrm{RhD}+$ \\
\hline 4676 & DHMi & partial D & $\mathrm{RhD}+$ \\
\hline \multicolumn{4}{|c|}{ c) Partial D by BLOODchip version 1.0 and weak D by serology (7) } \\
\hline 926 & DCS & partial D & weak D \\
\hline 1054 & DVI type II & partial D & weak D \\
\hline 1106 & DVI type II & partial D & weak D \\
\hline 1996 & DVI type IV & partial D & weak D \\
\hline 4654 & DVI type II & partial D & weak D \\
\hline 5317 & DVI type II & partial D & weak D \\
\hline 7632 & DVI type II & partial D & weak D \\
\hline \multicolumn{4}{|c|}{ d) Partial D by BLOODchip version 1.0 and $R h D-$ by serology (1) } \\
\hline 2253 & DIIIc & partial D & RhD- \\
\hline \multicolumn{4}{|c|}{ e) Weak D by BLOODchip version 1.0 and $R h D+$ by serology (6) } \\
\hline 615 & weak D type 2 & weak D & $\mathrm{RhD}+$ \\
\hline 3277 & $\begin{array}{l}\text { RHD (M295I)_possible ht Del / weak } \\
\text { type } 11\end{array}$ & Dweak D & $\mathrm{RhD}+$ \\
\hline 3586 & weak D type 2 & weak D & $\mathrm{RhD}+$ \\
\hline 4450 & weak D type 3 & weak D & $\mathrm{RhD}+$ \\
\hline 4978 & weak D type 4 & weak D & $\mathrm{RhD}+$ \\
\hline 7284 & weak D type 4 & weak D & $\mathrm{RhD}+$ \\
\hline \multicolumn{4}{|c|}{ f) Del by BLOODchip version 1.0 and RhD+ by serology (1) } \\
\hline 2874 & RHD (L153P) & Del & $\mathrm{RhD}+$ \\
\hline \multicolumn{4}{|c|}{ g) Del by BLOODchip version 1.0 and weak $D$ by serology (1) } \\
\hline 6769 & RHD (M295I)_Del & Del & weak D \\
\hline \multicolumn{4}{|c|}{ Discrepancies against BLOODchip version 1.0} \\
\hline Number & genotype (defined by BLOODchip) & BLOODchip & serology \\
\hline \multicolumn{4}{|c|}{ RhD+ by BLOODchip version 1.0 and weak D by serology (2) } \\
\hline 3694 & 'Apparently non-negative' & RHD & weak D \\
\hline 3695 & 'Apparently non-negative' & RHD & weak D \\
\hline
\end{tabular}

Eventually it may be economic to genotype every individual at birth. In that circumstance screening for inborn errors of metabolism (as suggested by many health authorities) could be coupled with both HLA and blood group genotyping. This information could then be highly useful to an individual as either a future patient or blood/organ donor during their lifetime.
Several genotyping platforms are now commercially available that gives the opportunity for blood banks to apply this technology for routine testing of their donors. This has a cost implication, and it is imperative that this is considered alongside the life-threatening circumstances of becoming alloimmunised as either a multi-transfused patient or an expectant mother. For this reason, studies involving the health-economic evaluation of the impact of mass-scale blood group genotyp- 
ing need to be conducted. There is no doubt that molecular genotyping has a significant edge over serology - several studies in addition to the Bloodgen project have proved this. The ball is now in the court of transfusion services to take these improvements and translate them into safer blood supplies and transfusion policies.

\section{Disclosure}

A. Martinez, D, Tejedor, M. López and E. Jiménez are employees of Progenika Biopharma S.A

\section{References}

$\checkmark 1$ Avent ND: Human erythrocyte antigen expression: its molecular bases. Br J Biomed Sci 1997;54:16-37.

2 Avent ND: Blood groups: Molecular genetic basis in Encyclopaedia of the Human Genome. London, Nature Publishing Group, 2003, vol A1034, pp 333343.

3 Cartron JP, Colin Y: Structural and functional diversity of blood group antigens. Transfus Clin Biol 2001;8:163-199.

4 Reid ME, Lomas-Francis C: The Blood Group Antigens Factsbook, 2nd ed. New York, Academic Press, 2004.

5 Daniels G: The molecular genetics of blood group polymorphism. Transpl Immunol 2005;14:143-153.

6 Lee S, Wu X, Reid M, Zelinski T, Redman C: Molecular basis of the kell (k1) phenotype. Blood 1995;85:912-916.

7 Bennett PR, Le Van Kim C, Colin Y, Warwick RM, Cherif-Zahar B, Fisk NM, Cartron JP: Prenatal determination of fetal RhD type by DNA amplification. N Engl J Med 1993;329:607-610.

$>8$ Wolter LC, Hyland CA, Saul A: Rhesus D genotyping using polymerase chain reaction. Blood 1993;82:1682-1683.

9 Avent ND, Martin PG, Armstrong-Fisher SS, Liu W, Finning KM, Maddocks D, Urbaniak SJ: Evidence of genetic diversity underlying Rh D-, weak $\mathrm{D}(\mathrm{Du})$, and partial D phenotypes as determined by multiplex polymerase chain reaction analysis of the RHD gene. Blood 1997;89:2568-2577.

10 Simsek S, Bleeker PM, von dem Borne AE: Prenatal determination of fetal RhD type. N Engl J Med 1994;330:795-796.

11 Westhoff CM: Molecular testing for transfusion medicine. Curr Opin Hematol 2006;13:471-475.

$\$ 12$ Le Van Kim C, Mouro I, Brossard Y, Chavinie J, Cartron JP, Colin Y: PCR-based determination of Rhc and RhE status of fetuses at risk of Rhc and RhE haemolytic disease. Br J Haematol 1994;88:193-195.

13 Avent ND, Martin PG: Kell typing by allele-specific PCR (ASP). Br J Haematol 1996;93:728-730.

14 Avent ND, Finning KM, Martin PG, Soothill PW: Prenatal determination of fetal blood group status. Vox Sang 2000;78(suppl 2):155-162.

$\checkmark 15$ Lo YM, Hjelm NM, Fidler C, Sargent IL, Murphy MF, Chamberlain PF, Poon PM, Redman CW, Wainscoat JS: Prenatal diagnosis of fetal RhD status by molecular analysis of maternal plasma. $\mathrm{N}$ Engl J Med 1998:339:1734-1738.

16 Faas BH, Beuling EA, Christiaens GC, von dem Borne AE, van der Schoot CE: Detection of fetal $\mathrm{RhD}$-specific sequences in maternal plasma. Lancet 1998;352:1196.
17 Finning KM, Martin PG, Soothill PW, Avent ND: Prediction of fetal D status from maternal plasma: introduction of a new noninvasive fetal RHD genotyping service. Transfusion 2002;42:1079-1085.

18 Denomme GA, Fernandes BJ: Fetal blood group genotyping. Transfusion 2007;47(1 suppl):64S-68S

19 Finning K, Martin P, Daniels G: The use of maternal plasma for prenatal RHD blood group genotyping. Methods Mol Biol 2009;496:143-157.

20 van der Schoot CE, Tax GH, Rijnders RJ, de Haas M, Christiaens GC: Prenatal typing of Rh and Kell blood group system antigens: the edge of a watershed. Transfus Med Rev 2003;17:31-44.

21 Avent ND: Recombinant technology in transfusion medicine. Curr Pharm Biotechnol 2000;1:117-135.

22 Avent ND: Large-scale blood group genotyping: clinical implications. Br J Haematol 2009;144:3-13. 23 Avent ND: Large scale blood group genotyping. Transfus Clin Biol 2007;14:10-15.

24 Legler TJ, Eber SW, Lakomek M, Lynen R, Maas JH, Pekrun A, Repas-Humpe M, Schroter W, Köhler M: Application of RHD and RHCE genotyping for correct blood group determination in chronically transfused patients. Transfusion 1999;39:852-855.

25 Reid ME, Rios M, Powell VI, Charles-Pierre D, Malavade V: DNA from blood samples can be used to genotype patients who have recently received a transfusion. Transfusion 2000;40:48-53.

26 Rozman P, Dove T, Gassner C: Differentiation of autologous ABO, RHD, RHCE, KEL, JK, and FY blood group genotypes by analysis of peripheral blood samples of patients who have recently received multiple transfusions. Transfusion 2000;40: 936-942.

27 Prager M: Molecular genetic blood group typing by the use of PCR-SSP technique. Transfusion 2007;47 (1 suppl):54S-59S.

28 Mouro I, Le Van Kim C, Rouillac C, van Rhenen DJ, Le Pennec PY, Bailly P, Cartron JP, Colin Y: Rearrangements of the blood group RHD gene associated with the DVI category phenotype. Blood 1994;83:1129-1135.

29 Avent ND, Liu W, Jones JW, Scott ML, Voak D, Pisacka M, Watt J, Fletcher A: Molecular analysis of $\mathrm{Rh}$ transcripts and polypeptides from individuals expressing the DVI variant phenotype: An RHD gene deletion event does not generate all DVIccEe phenotypes. Blood 1997;89:1779-1786.

30 Wagner FF, Gassner C, Müller TH, Schönitzer D, Schunter F, Flegel WA: Three molecular structures cause rhesus D category VI phenotypes with distinct immunohematologic features. Blood 1998;91: 2157-2168.

-31 Esteban R, Montero R, Flegel WA, Wagner FF, Subirana L, Parra R, Ribera A, Nogues N: The D category VI type 4 allele is prevalent in the Spanish population. Transfusion 2006;46:616-623.
2 Avent ND, Jones JW, Liu W, Scott ML, Voak D, Flegel WA, Wagner FF, Green C: Molecular basis of the D variant phenotypes DNU and DII allows localization of critical amino acids required for expression of Rh D epitopes epD3, 4 and 9 to the sixth external domain of the $\mathrm{Rh} \mathrm{D}$ protein. $\mathrm{Br} \mathrm{J}$ Haematol 1997;97:366-371.

33 Wagner FF, Frohmajer A, Ladewig B, Eicher NI, Lonicer CB, Müller TH, Siegel MH, Flegel WA: Weak D alleles express distinct phenotypes. Blood 2000;95:2699-2708.

34 Wagner FF, Gassner C, Müller TH, Schönitzer D, Schunter F, Flegel WA: Molecular basis of weak D phenotypes. Blood 1999;93:385-393.

35 Olsson ML, Smythe JS, Hansson C, Poole J, Mallinson G, Jones J, Avent ND, Daniels G: The Fy(x) phenotype is associated with a missense mutation in the Fy(b) allele predicting Arg89Cys in the Duffy glycoprotein. Br J Haematol 1998;103:1184-1191.

36 Tournamille C, Le Van Kim C, Gane P, Le Pennec PY, Roubinet F, Babinet J, Cartron JP, Colin Y: Arg89Cys substitution results in very low membrane expression of the Duffy antigen/receptor for chemokines in Fy(x) individuals. Blood 1998;92: 2147-2156.

37 Parasol N, Reid M, Rios M, Castilho L, Harari I, Kosower NS: A novel mutation in the coding sequence of the FY*B allele of the Duffy chemokine receptor gene is associated with an altered erythrocyte phenotype. Blood 1998;92:2237-2243.

38 Hult A, Hellberg A, Wester ES, Olausson P, Storry JR, Olsson ML: Blood group genotype analysis for the quality improvement of reagent test red blood cells. Vox Sang 2005;88:265-270.

39 Jones JW, Lloyd-Evans P, Kumpel BM: Quantitation of Rh D antigen sites on weak D and D variant red cells by flow cytometry. Vox Sang 1996;71:176183.

40 Daniels G, Poole G, Poole J: Partial D and weak D: Can they be distinguished? Transfus Med 2007;17: 145-146.

41 Flegel WA, Khull SR, Wagner FF: Primary anti-D immunization by weak D type 2 RBCs. Transfusion 2000;40:428-434.

42 Kormoczi GF, Gassner C, Shao CP, Uchikawa M, Legler TJ: A comprehensive analysis of Del types: Partial Del individuals are prone to anti-D alloimmunization. Transfusion 2005;45:1561-1567.

43 Vege S, Westhoff CM: Molecular characterization of GYPB and RH in donors in the American rare donor program. Immunohematology 2006;22:143147.

44 Moulds JM: Transfusion policies and practises in alloimmunised sickle cell patients. Haematologica 2009; (in press). 